NBER WORKING PAPER SERIES

\title{
EXCHANGE MARKET INTERVENTION UNDER ALTERNATIVE FORMS OF \\ EXOGENOUS DISTURBANCES
}

Stephen J. Turnovsky

Working Paper No. 1289

NATIONAL BUREAU OF ECONOMIC RESEARCH 1050 Massachusetts Avenue

Cambridge, MA 02138

March 1984

The research reported here is part of the NBER's research program in International Studies. Any opinions expressed are those of the author and not those of the National Bureau of Economic Research. 
NBER Working Paper 11289

March 1984

Exchange Market Intervention Under Alternative Forms of Exogenous Disturbances

\section{ABSTRACT}

This paper analyzes exchange market intervention in a stochastic model of a small open economy. The distinction is made between disturbances which are unanticipated and anticipated on the one hand, and those that are perceived as being transitory or permanent, on the other. The paper demonstrates how the appropriate form of exchange market intervention is sensitive to these aspects of the disturbances. of particular interest is the case of an unanticipated permanent disturbance, when output may be stabilized perfectly about its frictionless level by the use of a very simple class of intervention rules. The optimal rules in other cases are also discussed.

Stephen J. Turnovsky Department of Economics University of Illinois 1206 South Sixth Street Champaign, Illinois 61820

(217) $333-2354$ 


\section{INTRODUCTION}

With the freeing up of exchange rates during the last decade, interest has turned recently to questions related to exchange rate management. A particular issue which has been addressed by several economists is the determination of the optimal exchange rate regime, through intervention in the foreign exchange market. This literature has been developed within the context of small stochastic macro models, which in many respects are extensions of the well known framework developed by Poole (1970); see, e.g., Boyer (1979), Henderson (1979), Cox (1980), Roper and Turnovsky (1980), Frenkel and Aizenman (1982), and Turnovsky (1983). These models derive optimal intervention rules to stabilize some objective, typically domestic output, on the assumption that the stochastic disturbances impinging on the economy are all white noise; i.e., the stochastic disturbances are both unanticipated and transitory. One of the main conclusions to emerge is that the optimal policy can vary widely and depends critically upon the sources and relative magnitudes of the various stochastic disturbances.

In practice, of course, the stochastic disturbances facing an economy vary not only in origin, but also in nature. While some disturbances are unanticipated, others are expected; and while some may be transitory, others may be perceived as being permanent shifts. It would seem reasonable to expect that the appropriate form of exchange market intervention will depend upon these characteristics of the disturbances. To investigate this question is the purpose of this paper. Specifically, we extend the framework employed by Turnovsky (1983) to determine optimal exchange market intervention rules when the exogenous 
disturbances facing the economy may be of quite general form. In particular, we draw the distinction between disturbances which are:

(a) perceived as transitory or permanent, on the one hand, and (b) unanticipated or anticipated, on the other.

2. THE MODEL

To keep technical details down to a minimum, the simplest model will be used. Consider a small open economy which consumes and produces a single traded good. Assume also, that there is a single traded bond, with the domestic bond market being perfectly integrated with that in the rest of the world. Thus purchasing power parity (PPP) and uncovered interest parity (UIP) are assumed to hold.

Expressing all variables in deviation form, the model may be written as

$$
\begin{gathered}
p_{t}=q_{t}+e_{t} \\
m_{t}-p_{t}=\alpha_{1} y_{t}-\alpha_{2} r_{t}+u_{t} \alpha_{1}>0, \alpha_{2}>0 \\
r_{t}=\omega_{t}+e_{t+1, t}^{*}-e_{t} \\
m_{t}=\mu_{1} e_{t}+\mu_{2} e_{t+1, t}^{*}+\mu_{3} \omega_{t}+\mu_{4} q_{t} \\
y_{t}=\gamma\left(p_{t}-p_{t, t-1}^{*}\right)+v_{t}-\frac{\gamma_{t}^{*}, t-1}{I+n+r} r>0, n \geq 0
\end{gathered}
$$

while for any variable $\mathrm{x}_{t}$

$$
x_{s, t}^{*}=E_{t}\left(x_{s}\right)
$$

where 


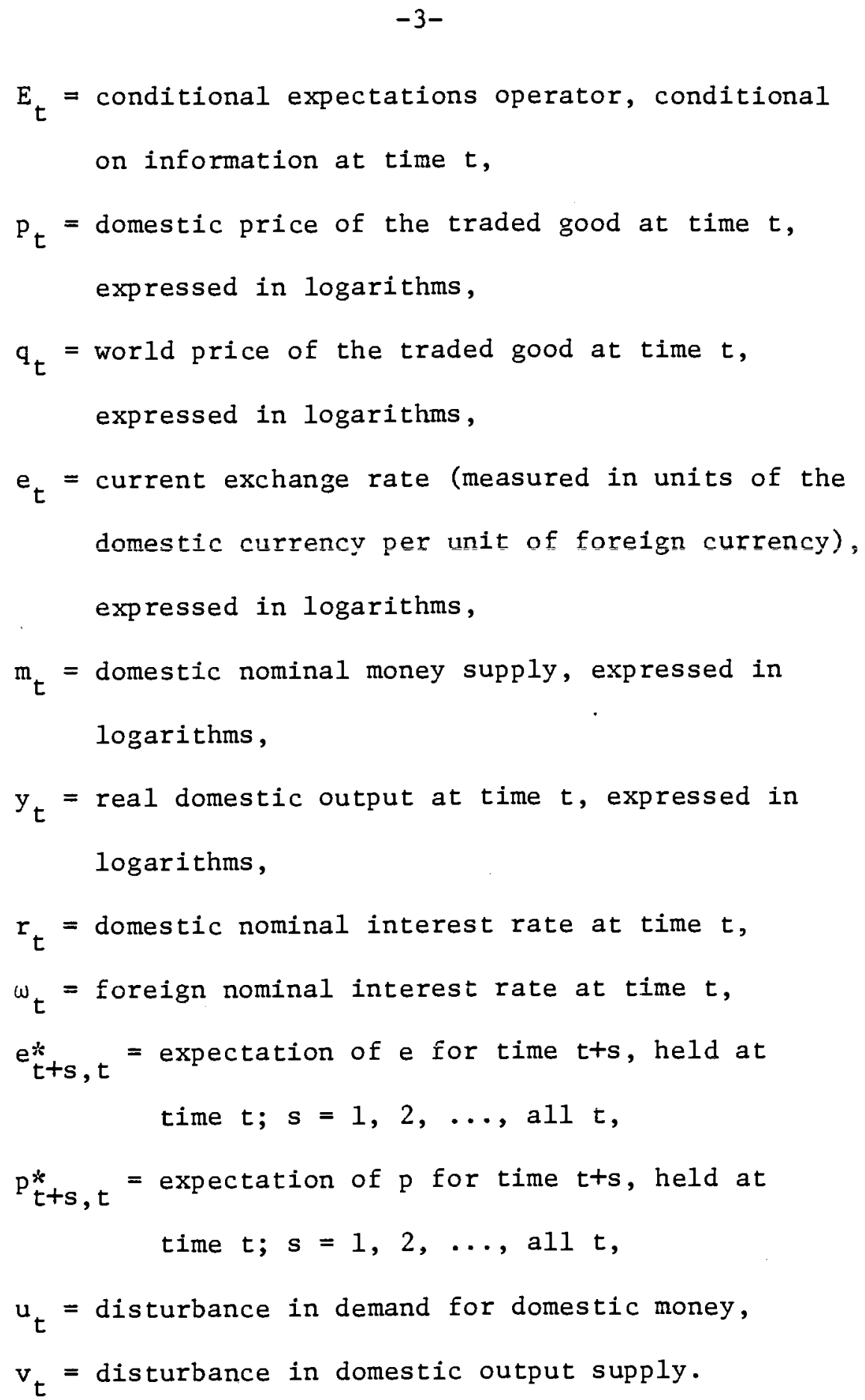

The first three equations of the model are quite standard. Given purchasing power parity, the domestic price of a freely traded commodity equals the price abroad multiplied by the exchange rate. Equation ( $1 a$ ) is just the logarthmic version of this relationship. Equation (1b) specifies domestic money market equilibrium, with the 
demand for money depending positively upon domestic income and negatively upon the domestic interest rate, and also upon an additive disturbance $u_{t} \cdot \underline{1 /}$ The assumption of perfectly capital market integration is embodied in the interest rate parity condition (1c).

The remaining two equations are somewhat less standard and require further comment. Equation (1d) specifies a money supply rule. We assume that all changes in the domestic money supply are brought about by the accumulation or decumulation of foreign reserves through intervention in the foreign exchange market, with the central bank's liabilities created against the purchase of domestic assets remaining fixed. The underlying notion behind (1d) is that the intervention authority has current information on all the financial variables, $e_{t}$, $r_{t}$, and $\omega_{t}$, as well as the domestic price level $p_{t}$. Thus we postulate

$$
m_{t}=v_{1} e_{t}+v_{2} r_{t}+v_{3} w_{t}+v_{4} p_{t}
$$

Using the PPP condition. ( $1 a$ ) and the UIP condition (1c), this equation is equivalent to the form (1d). The coefficients $\mu_{i}$ are the policy parameters and the optimal intervention policy is to choose them so as to optimize an objective, yet to be specified. Note that the observability of $e_{t}$ and $p_{t}$, together with PPP, implies the observability of the foreign price level $q_{t}$. In contrast to most of the existing intervention literature which restricts the rule to depend upon only the current exchange rate, (1d) allows it to depend upon observations of other variables, which are observable with virtually equal frequency as exchange rates, thereby enabling the intervention authority to base its decisions on a wider information set. 
The supply of domestic output is specified by (le). This relationship postulates the deviation in output from its full employment level to depend upon the unanticipated component of the current domestic price of output, a current productivity disturbance and the previous period's expectation of the current productivity disturbance. This specification can be derived from the one period wage contract models of Fischer (1977) and Gray (1976) and is given in the Appendix. Using this specific framework, $\gamma$ is a function of the labor exponent in the firm's Cobb-Douglas production function, while $\mathrm{n}$ is the supply elasticity of labor. As shown in the derivation, the expectation of the productivity disturbance enters the supply function through its influence on the wage contract. Under the commonly made assumption that these disturbances are independently distributed over time, this expectational term is just zero. Finally, equation (1f) describes the rationality of expectations. There are four additive disturbances in the model, two of domestic origin $\left(u_{t}, v_{t}\right)$ and two of foreign origin $\left(q_{t}, \omega_{t}\right)$. The existing literature treats these as being purely random variables, which are independently distributed over time. They are therefore unanticipated and transitory. In this analysis, we do not restrict them in this way. All we shall assume is that they be exogenous to the small country under consideration.

3. SOLUTION OF MODEL

In order to solve the model, we first take conditional expectations of (la)-(1e). This is most conveniently done by considering the expectations for an arbitrary date $i$, formed at some initial date o 


$$
\begin{gathered}
p_{i, o}^{*}=q_{i, o}^{*}+e_{i, o}^{*} \quad i=1,2, \ldots \\
m_{i, o}^{*}-p_{i, o}^{*}=\alpha_{1} y \underset{i, o}{*}-\alpha_{2} r_{i, o}^{*}+u_{i, o}^{*} \\
r_{i, o}^{*}=w_{i, o}^{*}+e_{i+1, o}^{*}-e_{i, o}^{*} \\
m_{i, o}^{*}=\mu_{1} e_{i, o}^{*}+\mu_{2} e_{i+1, o}^{*}+\mu_{3} \omega_{i, o}^{*}+\mu_{4} q_{i, o}^{*} \\
y_{i, o}^{*}=\left(\frac{1+n}{1+n+\gamma}\right) v_{i, o}^{*}
\end{gathered}
$$

Substituting (2a), (2c), (2d) and (2e) inta (2b) gives rise to the following first order difference equation in expectations $e_{i, o}^{*}$

$$
\left(\alpha_{2}+\mu_{2}\right) e_{i+1,0}^{*}-\left(1-\mu_{1}+\alpha_{2}\right) e_{i, o}^{*}=z_{i, o}^{*} \quad i=1,2, \ldots
$$

where

$$
z_{i, 0}^{*} \equiv u_{i, 0}^{*}+\frac{\alpha_{1}(1+n)}{1+n+\gamma} v_{i, 0}^{*}+\left(1-\mu_{4}\right) q_{i, o}^{*}-\left(\alpha_{2}+\mu_{3}\right) \omega_{i, 0}^{*}
$$

and we define

$$
z_{t} \equiv u_{t}+\frac{\alpha_{1}(1+n)}{1+n+\gamma} v_{t}+\left(1-\mu_{4}\right) q_{t}-\left(\alpha_{2}+\mu_{3}\right) \omega_{t}
$$

The stable solution to this equation is

$$
\begin{gathered}
e_{i, o}^{*}=-\frac{1}{1-\mu_{1}+\alpha_{2}}\left[\sum_{j=0}^{\infty} z_{i+j, o}^{*}\left(\frac{a_{2}+\mu_{2}}{1-\mu_{1}+\alpha_{2}}\right)^{j}\right] \text { if }\left|\frac{1-\mu_{1}+\alpha_{2}}{\alpha_{2}+\mu_{2}}\right|>1 \quad i=1,2, \ldots \\
\quad e_{i, o}^{*}=\frac{1}{\alpha_{2}+\mu}\left[\sum_{j=0}^{\infty} z_{i-1-j, 0}^{*}\left(\frac{1-\mu_{1}+\alpha_{2}}{\alpha_{2}+\mu_{2}}\right)^{j}\right] \text { if }\left|\frac{1-\mu_{1}+\alpha_{2}}{\alpha_{2}+\mu_{2}}\right|<1 \quad \text { (5b) }
\end{gathered}
$$

where in $(5 b), z_{i-j, o}^{*}=z_{i-j}$ for $i \geq j . \underline{2 /}$ Note that the form of the stable solution depends critically upon the monetary authority's intervention parameters $\mu_{1}$ and $\mu_{2} \cdot \underline{3}$ / If the degree of intervention is such as 
to render the system unstable (in the sense of the eigenvalue $\left(1-\mu_{1}+\alpha_{2}\right) /$ $\left(\alpha_{2}+\mu_{2}\right)$ lying outside the unit circle) then the stable solution is such that the expectation of the exchange rate for time $i$, formed at time 0 is a function of the discounted sum of expectations for all periods into the future. This is the conventional form of rational expectations solutions and arises in the case of a perfectly flexible exchange rate $\left(i_{i}=0\right)$. On the other hand, if the degree of intervention is such as to render the system stable (in the sense that the eigenvalue lies within the unit circle), then the stable solution for expectations is a backward function of past expectations, where we assume that predictions formed at time 0 for current or past values are correct. 4 /

To obtain the solutions for the key endogenous variables $y_{t}$, $p_{t}$, and $e_{t}$ we may reduce the basic set of equations (1a)-(1e) to the following matrix equation

$$
\left(\begin{array}{ccc}
0 & 1 & -1 \\
\alpha_{1} & 1 & \alpha_{2}-\mu_{1} \\
1 & -\gamma & 0
\end{array}\right)\left(\begin{array}{l}
y_{t} \\
p_{t} \\
e_{t}
\end{array}\right)=\left(\begin{array}{l}
q_{t} \\
-u_{t}+\left(\mu_{2}+\alpha_{2}\right) e_{t+1, t}^{*}+\left(\mu_{3}+\alpha_{2}\right) \omega_{t}+\mu_{4} q_{t} \\
v_{t}-\gamma\left[e_{t, t-1}^{*}+q_{t, t-1}^{*}+v_{t, t-1}^{*} /(1+n+\gamma)\right]
\end{array}\right)
$$

It is clear that the choice of conditioning date, 0 , was made for notational convenience and that analogous solutions to (5) hold with respect to expectations formed at any arbitrary time $t$. Specifically, the relevant expressions appearing in (6) are

$$
\begin{gathered}
e_{t+1, t}^{*}=-\frac{1}{1-\mu_{1}+\alpha_{2}}\left[\sum_{j=0}^{\infty} z_{t+1+j, t}^{*}\left(\frac{\alpha_{2}+\mu_{2}}{1-\mu_{1}+\alpha_{2}}\right)^{j}\right] \text { if }\left|\frac{1-\mu_{1}+\alpha_{2}}{\alpha_{2}+\mu_{2}}\right|>1 \\
e_{t+1, t}^{*}=\frac{1}{\alpha_{2}+\mu_{2}}\left[\sum_{j=0}^{\infty} z_{t-j, t}^{*}\left(\frac{1-\mu_{1}+\alpha_{2}}{\alpha_{2}+\mu_{2}}\right)^{j}\right] \text { if }\left|\frac{1-\mu_{1}+\alpha_{2}}{\alpha_{2}+\mu_{2}}\right|<1 \\
p_{t, t-1}^{*}=e_{t, t-1}^{*}+q_{t, t-1}^{*}
\end{gathered}
$$


In our analysis below, we shall deal with two important cases. First, we shall assume that the expectations of the composite variable $z_{t}$, formed at time $t$ say, are uniform throughout all future periods. Formally, this is described by

$$
z_{t+i, t}^{*}=z_{t}^{*} \text { say }, i=1,2, \ldots
$$

and characterizes a situation where the disturbance in $z$ is expected to be permanent. In this case, the stable solution for exchange rate expectations (7a) and (7b) both simplify to $5 /$

$$
e_{t+1, t}^{*}=\frac{-z_{t}^{*}}{1-\mu_{1}-\mu_{2}} \quad t=1,2, \ldots
$$

The second, is where at time $t$ say, a transitory disturbance in $z$ for the next period $t+1$ is expected; i.e.,

$$
z_{t+1, t}^{*} \neq 0 ; z_{t+i, t}^{*}=0 \quad i=2,3, \ldots
$$

In this case the stable solution for exchange rate expectations is $6 /$

$$
e_{t+1, t}^{*}=\frac{-z_{t+1, t}^{*}}{1-\mu_{1}+\alpha_{2}} \quad t=1,2, \ldots
$$

In order to determine the optimal degree of intervention, we must introduce a criterion function. In the literature, two alternative criteria are frequently adopted. The first of these is the conventional one of minimizing the variance of domestic output about its constant long-run mean. The second, introduced by Barro (1976) and Gray (1976) is the minimization of the variance of output about its full information frictionless level. This latter quantity, denoted by $y_{t}^{f}$, is defined as being the level of output that would prevail if wages were fully flexible and not preset by a contract. In the present model, it is well known that 


$$
\dot{y}_{t}^{f}=\left(\frac{1+n}{1+n+\gamma}\right) v_{t}
$$

so that $y_{t}^{f}$ fluctuates to some degree with the productivity disturbance embodied in $v_{t}$. While both criteria are of interest, and indeed identical with respect to all but the supply disturbances, we shall focus on the latter, which recently has been increasingly adopted in the literature. $7 /$

Solving the matrix equation (6), we find that the short-run solutions for the endogenous variables $y_{t}, p_{t}$ and $e_{t}$ are given by

$$
\begin{gathered}
y_{t}=y_{t}^{f}+\frac{\gamma}{\Delta}\left[-z_{t}+\frac{\left(1+\alpha_{2}-\mu_{1}\right)}{1+n+\gamma}\left(v_{t}-v_{t, t-1}^{*}\right)+\left(1+\alpha_{2}-\mu_{1}\right)\left(q_{t}-q_{t, t-1}^{*}\right)\right. \\
\left.+\left(\mu_{2}+\alpha_{2}\right) e_{t+1, t}^{*}-\left(1+\alpha_{2}-\mu_{1}\right) e_{t, t-1]}^{*}\right] \\
p_{t}=e_{t, t-1}^{*}+q_{t, t-1}^{*}+\frac{1}{\Delta}\left[-z_{t}-\frac{\alpha_{1} \gamma}{1+n+\gamma}\left(v_{t}-v_{t, t-1}^{*}\right)+\left(1+\alpha_{2}-\mu_{1}\right)\left(q_{t}-q_{t, t-1}^{*}\right)\right. \\
\left.+\left(\mu_{2}+\alpha_{2}\right) e_{t+1, t}^{*}-\left(1+\alpha_{2}-\mu_{1}\right) e_{t, t-1}^{*}\right] \\
e_{t}=\frac{1}{\Delta}\left[-z_{t}-\frac{\alpha_{1} \gamma}{1+n+\gamma}\left(v_{t}-v_{t, t-1}^{*}\right)-\alpha_{1} \gamma\left(q_{t}-q t_{t, t-1}^{*}\right)\right. \\
\left.\quad+\left(\mu_{2}+\alpha_{2}\right) e_{t+1, t}^{*}+\alpha_{1} \gamma e_{t, t-1}^{*}\right]
\end{gathered}
$$

where $\Delta \equiv 1-\mu_{1}+\alpha_{2}+\alpha_{1} \gamma$ and $e_{t+1, t}^{*}$ is given by (7) above. This solution expresses the endogenous variables in terms of the exogenous composite disturbance $z_{t}$ defined in $\left(4^{\prime}\right), v_{t}$, and $q_{t}$, together with the expectations for exchange rates $e_{t+1, t}^{*}$ and $e_{t, t-1}^{*}$ given by (7) above, the expectation of the productivity disturbance $v_{t, t-1}^{*}$, and the expectation of the foreign price disturbance $q_{t, t-1}^{*}$, the latter two which we assume to be exogenous. It is evident that the degree of exchange market intervention as described by the parameters $\mu_{i}$ influences 
the behavior of the economy in two ways. First, it affects how expectations of future exchange rates respond to expectations about the exogenous disturbances embodied in $z_{t+j, t}^{*}$; secondly, it affects how the current stochastic disturbances $u_{t}, v_{t}, q_{t}$, and $w_{t}$ impinge on the domestic economy .

\section{OPTIMAL EXCHANGE MARKET INTERVENTION}

Equations (6) and (4) form the basis for our analysis of optimal exchange market intervention. The assumptions we have made are sufficiently general to enable us to analyze intervention policies under quite arbitrary assumptions regarding the nature of the exogenous disturbances impinging on the economy. We shall restrict our attention to distinguishing between disturbances which are: (a) unanticipated or anticipated; (b) perceived as being transitory or permanent. Note that in making the latter distinction, we can do so only in terms of the perception at the time they occur. It is quite possible that a disturbance which when it occurs at time $t$ say is expected to be permanent, is in fact reversed during the next period. Initially, it will have been perceived as being permanent, although in fact it will turn out to be only transitory.

(a) Unanticipated, Perceived Transitory, Disturbances

Consider the situation in which all disturbances are independently distributed over time; they are therefore unanticipated and perceived as being transitory. As noted above, this case has been extensively discussed in the literature and hence our treatment can be brief.

This case is parameterized by setting $q_{t+1, t}^{*}=0, v_{t+1, t}^{*}=0$, $z_{t+j, t}^{*}=0$; for all $j$, t. It therefore follows from (6) that

$$
e_{t+1, t}^{*}=0
$$


with the corresponding solution for output being

$$
y_{t}=y_{t}^{f}+\frac{\gamma}{\Delta}\left[-u_{t}+\left(\frac{\Delta}{1+n+\gamma}-\alpha_{1}\right) v_{t}+\left(\alpha_{2}+\mu_{3}\right) \omega_{t}+\left(\alpha_{2}+\mu_{4}-\mu_{1}\right) q_{t}\right]
$$

Note that the solution of output is independent of the intervention parameter $\mu_{2}$. This is because for white noise disturbances $e_{t+1, t}^{*}=0$. It is evident from (15) that the values of the optimal policy parameters, $\hat{\mu}_{i}$ say, which minimize the variance of output around $y_{t}^{f}$ can be attained recursively. First, given $\hat{\mu}_{1}$, the optimal values for $\hat{\mu}_{3}$ and $\hat{\mu}_{4}$ are simply obtained by setting the coefficients of $\omega_{t}$ and $q_{t}$ in (15) to zero, namely.

$$
\begin{gathered}
\hat{\mu}_{3}=-\alpha_{2} \\
\hat{\mu}_{4}=-\alpha_{2}+\hat{\mu}_{1}
\end{gathered}
$$

It therefore follows that by appropriate intervention in response to $\omega_{t}$ and $\mathrm{q}_{\mathrm{t}}$ it is possible to stabilize domestic output perfectly against all foreign disturbances. Substituting (16a) and (16b) into (15) and taking expectations, the variance of output about $y_{t}^{f}$, denoted by $\sigma_{y}^{2}$, given the choice of $\mu_{3}$ and the conditionally optimal choice of $\mu_{4}$ is

$$
\sigma_{y}^{2}=\frac{\gamma^{2}}{\Delta^{2}}\left[\sigma_{u}^{2}+\left(\frac{\Delta}{1+n+\gamma}-\alpha_{1}\right)^{2} \sigma_{v}^{2}\right]
$$

The remaining problem is to choose $\mu_{1}$ to minimize (17). Carrying out the differentiation leads to the optimality condition

$$
\hat{\mu}_{1}=1+\alpha_{2}-\alpha_{1}(1+n)-\frac{(1+n+\gamma) \sigma_{u}^{2}}{\alpha_{1} \sigma_{v^{r}}^{2}}
$$

and combining (16b) and (18) we find

$$
\hat{\mu}_{4}=1-\alpha_{1}(1+n)-\frac{(1+n+y) \sigma_{u}^{2}}{\alpha_{1} \sigma_{v}^{2}}
$$


Thus with $e_{t+1, t}^{*}=0$, the optimal intervention rule can be expressed as $\mathrm{m}_{\mathrm{t}}=\left[1+\alpha_{2}-\alpha_{1}(1+n)-\frac{(1+n+\gamma) \sigma_{u}^{2}}{\alpha_{1} \sigma_{v}^{2}}\right] e_{t}-\alpha_{2} \omega_{t}+\left[1-\alpha_{1}(1+n)-\frac{(1+n+\gamma) \sigma_{u}^{2}}{\alpha_{1} \sigma_{v}^{2}}\right] q_{t}$

which, using the PPP and UIP conditions becomes

$$
\mathrm{m}_{\mathrm{t}}=\left[1-\alpha_{1}(1+\mathrm{n})-\frac{(1+\mathrm{n}+\gamma) \sigma_{\mathrm{u}}^{2}}{\alpha_{1} \sigma_{\mathrm{v}}^{2}}\right] \mathrm{p}_{\mathrm{t}}-\alpha_{2} \mathrm{r}_{\mathrm{t}}
$$

Thus the optimal intervention policy can be conveniently expressed in terms of responses in the domestic money supply to movements in:

(1) the domestic nominal interest rate; and (ii) the domestic price level. A one percentage point rise in the former should be met by a $\alpha_{2}$ percent reduction in the money supply. A one percent rise in the domestic price level should be accommodated by a less than one percent rise in the nominal money supply. The adjustment depends positively upon the variance of the domestic real disturbance and inversely on the variance of the domestic monetary disturbance. Substituting for the optimal $\mu_{1}$ into (18) we find that the optimized variance of output is generally strictly positive. One is not able to stabilize output perfectly about its frictionless level, unless either $u_{t} \equiv 0$ or $v_{t} \equiv 0$. Further discussion of the present case is given by Turnovsky (1984).

\section{(b) Unanticipated, Perceived Permanent, Disturbances}

Suppose that the disturbances occurring at time $t$ were previously (i.e., at time t-1) unanticipated, but having occurred are expected to be permanent. This is parameterized by assuming $\mathrm{q}_{\mathrm{t}, \mathrm{t}-1}^{\star}=\mathrm{v}_{\mathrm{t}, \mathrm{t}-1}^{*}=\mathrm{z}_{\mathrm{t}+\mathrm{j}, \mathrm{t}-1}=0$, for all $j, z_{t+j, t}^{\star}=z_{t}$, for all $j$. It thus follows from ( $\left.\dot{9}\right)$ that exchange rate expectations are generated by 


$$
\begin{gathered}
-13- \\
e_{t, t-1}^{*}=0 \\
e_{t+1, t}^{*}=\frac{-z_{t}}{1-\mu_{1}-\mu_{2}}
\end{gathered}
$$

The corresponding solution for real output, obtained by substituting $(22 a),(22 b)$ into $(13 a)$, is

$$
y_{t}=y_{t}^{f}+\frac{\gamma\left(1+\alpha_{2}-\mu_{1}\right)}{\Delta}\left[\frac{v_{t}}{1+n+\gamma}-\frac{z_{t}}{1-\mu_{1}-\mu_{2}}+q_{t}\right]
$$

The optimal intervention policy follows immediately upon inspection of (23). It is simply to set $\mu_{1}=\left(1+\alpha_{2}\right)$, allowing the remaining parameters $\mu_{2}, \mu_{3}, \mu_{4}$, to be set arbitrarily, thereby yielding a very general optimal policy rule

$$
m_{t}=\left(1+\alpha_{2}\right) e_{t}+\mu_{2} e_{t+1, t}^{*}+\mu_{3} \omega_{t}+\mu_{4} q_{t}
$$

Intervening in accordance with this rule ensures that $y_{t}$ is stabilized perfectly at its frictionless level, irrespective of the source of the stochastic disturbances.

This is an extremely powerful result and contrasts dramatically with the more familiar case where disturbances are unanticipated and transitory. In that situation optimal intervention depends upon a knowledge of the relative variances of the domestic disturbances and does not in general succeed in stabilizing income perfectly. For disturbances which are perceived as being permanent, however, any intervention rule, which includes the leaning with the wind component $\mathrm{m}=\left(1+\alpha_{2}\right) \mathrm{e}$ will stabilize income perfectly about its frictionless level.

The key to understanding the economic intuition underlying this result is the domestic money market. Combining (1a), (1b) and (1c) we have 


$$
m_{t}=q_{t}+e_{t}+\alpha_{1} y_{t}-\alpha_{2}\left[w_{t}+e_{t+1, t}^{*}-e_{t}\right]+u_{t}
$$

If the domestic monetary authorities intervene in accordance with the rule (24), it is seen from (22a) and (22b) that

$$
\begin{gathered}
e_{t, t-1}^{*}=0 \\
e_{t+1, t}^{*}=\frac{1}{\alpha_{2}+\mu_{2}}\left[u_{t}+\frac{\alpha_{1}(1+n)}{1+n+\gamma} v_{t}-\left(\alpha_{2}+\mu_{3}\right) \omega_{t}+\left(1-\mu_{4}\right) q_{t}\right]
\end{gathered}
$$

Being unanticipated, prior to the disturbance, the exchange rate is not expected to change. However, since the disturbance having occurred is expected to be permanent, the exchange rate in the following period is expected to adjust in response to the disturances in $u_{t}, v_{t}, \omega_{t}$, and $q_{t}$. The adjustment in the domestic interest rate which is the immediate consequence of this is precisely such as to render the excess demand for nominal money balances independent of the disturbances $u_{t}$, $\omega_{t}$, and $q_{t}$. This can be seen by substituting (26b) and (24) into (25) to yield

$$
\begin{gathered}
\left(1+\alpha_{2}\right) e_{t}+\mu_{3} \omega_{t}+\mu_{4} q_{t}=q_{t}+e_{t}+\alpha_{1} y_{t}-\left[u_{t}+\frac{\alpha_{1}(1+n)}{1+n+\gamma} v_{t}-\left(\alpha_{2}+\mu_{3}\right) \omega_{t}+\left(1-\mu_{4}\right) q_{t}\right] \\
-\alpha_{2}\left(\omega_{t}-e_{t}\right)+u_{t}
\end{gathered}
$$

It is clear from this equation that whatever values of $\mu_{2}, \mu_{3}, \mu_{4}$, are chosen, the expected exchange rate, given by the term in parentheses, simply adjusts appropriately. Cancelling terms, equation (27) reduces to

$$
y_{t}=\left(\frac{1+n}{1+n+\gamma}\right) v_{t}=y_{t}^{f}
$$

thereby stabilizing income about its frictionless level. 
The fact that $\mu_{2}, \mu_{3}$, and $\mu_{4}$ may be set arbitrarily means that the optimal intervention rule can be specified in a number of convenient ways. Perhaps the most convenient is to set $\mu_{2}=\mu_{3}=\mu_{4}=0$, in which case (24) reduces to the simple leaning with the wind rule

$$
\mathrm{m}_{\mathrm{t}}=\left(1+\alpha_{2}\right) \mathrm{e}_{\mathrm{t}}
$$

However, setting $\mu_{2}=\mu_{3}=0, \mu_{4}=\left(1+\alpha_{2}\right)$, enables it to be expressed in the form

$$
\mathrm{m}_{\mathrm{t}}=\left(1+\alpha_{2}\right) \mathrm{p}_{\mathrm{t}}
$$

in which case the optimal policy is specified in terms of a more than proportionate accommodation of the money supply to the domestic price level. Also, setting $\mu_{2}=\mu_{3}=-\left(1+\alpha_{2}\right), \mu_{4}=0$, the rule can be expressed in terms of the domestic interest rate

$$
m_{t}=-\left(1+\alpha_{2}\right) r_{t}
$$

In all cases, the perfect stabilization of domestic output is due to the appropriate adjustment of the expected exchange rate. Which is the most convenient form will presumably depend upon the availability and reliability of the necessary information.

It is worth noting that in some cases, output can be stabilized optimally using yet another rule. Recall (23) and consider the term in parentheses, which after substituting for $z_{t}$ is given by

$$
\frac{-u_{t}}{1-\mu_{1}-\mu_{2}}+\frac{v_{t}}{1+n+\gamma}\left[1-\frac{\alpha_{1}(1+n)}{1-\mu_{1}-\mu_{2}}\right]-\frac{\left(\alpha_{2}+\mu_{3}\right) \omega_{t}}{1-\mu_{1}-\mu_{2}}+\left(\frac{\mu_{4}-\mu_{1}-\mu_{2}}{1-\mu_{1}-\mu_{2}}\right) q_{t}
$$


Setting $\mu_{3}=-\alpha_{2}$, and $\mu_{4}=\mu_{1}+\mu_{2}$ one can eliminate the variance in output due to $\omega_{t}$ and $q_{t}$. However, in general one cannot eliminate completely the total variance due to domestic disturbances, unless of course one sets $\mu_{1}=\left(1+\alpha_{2}\right)$, as discussed above. But if $u_{t} \equiv 0\left(\sigma_{u}^{2}=0\right)$, so that the only domestic disturbances are due to productivity disturbances, $y_{t}$ can be stabilized about $y_{t}^{f}$ by adopting the rule

$$
m_{t}=\left[1-\alpha_{1}(1+n)\right] p_{t}-\alpha_{2} r_{t}
$$

This policy is identical to (21), derived in the case of transitory shocks, when one sets $\sigma_{\mathrm{u}}^{2}=0$ in the latter.

The economic rationale for this result follows from a consideration of both the domestic money market and domestic supply function. Substituting (29) into (25) and simplifying (noting that expectations at tiem t-1 are zero), these two relationships simplify to respectively

$$
\begin{gathered}
p_{t}=\frac{-y_{t}}{1+n} \\
y_{t}=\gamma p_{t}+v_{t}
\end{gathered}
$$

The solution for $y_{t}$ is again given by (28). The productivity shock $v_{t}$ as it impinges on the domestic supply function, generates fluctuations in output which exceed those of the frictionless economy. If by intervening in the exchange market, the price level can be made to bear part of the adjustment, the fluctuations in output can be dampened down to the appropriate level.

In the two cases we have thus far considered, we have assumed that the private sector in its perception as to whether the disturbances which have occurred are permanent or transitory. Of course in due course 
they may prove to be wrong, but our assumption is that agents can form a subjective characterization of them. In fact, of course, they may be unable to decide whether a disturbance which has occurred represents a permanent shift or is simply a transitory shock. One way to formalize this is to assign a probability to these states. In this case the expected exchange rate formed at time $t$ becomes

$$
e_{t+1, t}^{*}=\frac{-\theta z_{t}}{1-\mu_{1}-\mu_{2}}
$$

where $\theta$ is the probability of the disturbance being permanent. This expression is derived by taking an average of (14) and (22b), each weighted by their respective probabilities $1-\theta, \theta$. The analysis can be carried out as before, with (22b) being replaced by (30). However, if $\theta<1$, it can be shown that in general perfect stabilization of $y_{t}$ about $y_{t}^{f}$ is not possible. $\underline{9}$

(c) Random Walks

Section $4(\mathrm{~b})$ has dealt with the case of once-and-for-all disturbances, initially unanticipated, but ex post perceived as being permanent. This is closely related to the important case where the disturbances follow random walks. In this case the relevant random variables appearing in (14) are generated by

$$
\begin{aligned}
& z_{t}=z_{t-1}+\eta_{z t} \\
& v_{t}=v_{t-1}^{\cdot}+\eta_{v t} \\
& q_{t}=q_{t-1}+\eta_{q t}
\end{aligned}
$$

where the $\eta_{i t}$ are independently distributed random variables having zero means and finite variances. Any change in $\eta_{i t}$ is unanticipated at time 
$t-1$, but having occurred, is perceived at time $t$ as being permanent and becomes part of all future expectations formed at that time. For this process, the expectations of the exchange rate formed at time $t$ and $t-1$ are respectively

$$
e_{t+1, t}^{*}=\frac{-z_{t}}{1-\mu_{1}-\mu_{2}} ; e_{t, t-1}^{*}=\frac{-z_{t-1}}{1-\mu_{1}-\mu_{2}}
$$

and substituting (31) into (14a), the solution for output is given by

$$
y_{t}=y_{t}^{f}+\frac{\gamma\left(1+\alpha_{2}-\mu_{1}\right)}{\Delta}\left[-\frac{n_{z t}}{1-\mu_{1}-\mu_{2}}+\frac{\eta_{v t}}{1+n+\gamma}+\eta_{q t}\right]
$$

This is virtually identical to (23), the only difference being that the disturbance terms $z_{t}$, $v_{t}$, and $q_{t}$ are replaced by their changes, $\eta_{t}$ ' $\eta_{v t}$, $\eta_{q t}$, reflecting the non-stationarity of the random walk process. The optimal policy is again given by (25) and the explanation given previously continues to apply.

\section{(d) Anticipated Disturbances}

Thus far, all disturbances have been assumed to be unanticipated. Sometimes, however, they may be known in advance and as an example consider the following. Suppose that at time $t-1$ agents perfectly anticipate all disturbances for the next period; i.e., $q_{t, t-1}^{*}=q_{t}, v_{t, t-1}^{*}=v_{t}, z_{t, t-1}^{*}=z_{t}$. However, although they know the magnitudes of these shocks for the next period, they do not know whether they are transitory shocks or permanent shifts. Suppose that at time $t-1$, before the disturbances occur, agents assign a probability $\theta_{t-1}$ to the event that they are permanent, so that $\left(1-\theta_{t-1}\right)$ equals the probability that they perceive it as being transitory. The expected exchange rate for time $t$ formed at time $t-1$ is therefore 


$$
e_{t, t-1}^{*}=-\left[\frac{\theta_{t-1}}{1-\mu_{1}-\mu_{2}}+\frac{\left(1-\theta_{t-1}\right)}{1-\mu_{1}+\alpha_{2}}\right] z_{t}
$$

This equation is simply an average of (9), (11) at $t-1$ weighted by their respective probabilities. Substituting (33) into (13a) we find that the solution for output becomes

$$
y_{t}=y_{t}^{f}+\frac{y}{\Delta}\left(\mu_{2}+\alpha_{2}\right)\left[e_{t+1, t}^{*}+\frac{\theta_{t-1} z_{t}}{1-\mu_{1}-\mu_{2}}\right]
$$

It follows from (34) that if the policy maker sets $\mu_{2}=-\alpha_{2}$, then

$$
y_{t}=y_{t}^{f} \quad \text { for all } t
$$

irrespective of the private sector's perception of the disturbances at time $t$ (after they have occurred) as reflected in $e_{t+1, t}^{*}$ Thus the monetary rule

$$
m_{t}=\mu_{1} e_{t}-\alpha_{2} e_{t+1, t}^{*}+\mu_{3} w_{t}+\mu_{4} q_{t}
$$

where $\mu_{1}, \mu_{3}, \mu_{4}$, are arbitrary will stabilize output perfectly at its frictionless level.

The reason is as follows. If the shocks are perfectly anticipated, the supply function (le) becomes

$$
y_{t}=\gamma\left(e_{t}-e_{t, t-1}^{*}\right)+\left(\frac{1+n}{1+n+\gamma}\right) v_{t}=\gamma\left(e_{t}-e_{t, t-1}^{*}\right)+y_{t}^{f}
$$

Thus output will be stabilized at $y_{t}^{f}$ if and only if $e_{t, t-l}^{*}=e_{t}$. However, in general the current exchange rate $e_{t}$ is influenced by $e_{t+1, t}^{*}$ through the interest rate in the money market equilibrium relationship. By adopting an intervention rule with $\mu_{2}=-a_{2}$, the monetary authority eliminates the effect of the expected future exchange rate from the money market condition, reducing it to 


$$
\alpha_{1} y_{t}-\left(1+\alpha_{2}-\mu_{1}\right) e_{t}+\left(\alpha_{2}+\mu_{3}\right) \omega_{t}+\left(1-\mu_{4}\right) q_{t}-u_{t}=0
$$

The effect of setting $\mu_{2}=-\alpha_{2}$ means that $e_{t, t-1}^{*}$ is the same whether the disturbances are initially perceived as being permanent or transitory, so that

$$
e_{t, t-1}^{*}=\frac{-z t}{1-\mu_{1}+\alpha_{2}}=\frac{-1}{1-\mu_{1}+\alpha_{2}}\left[u_{t}+\alpha_{1} y_{t}^{f}-\left(\alpha_{2}+\mu_{3}\right) \omega_{t}+\left(1-\mu_{4}\right) q_{t}\right]
$$

Combining (36) and (37), the money market equilibrium condition (36) reduces' further to

$$
\alpha_{1}\left(y_{t}-y_{t}^{f}\right)=\left(1+\alpha_{2}-\mu_{1}\right)\left(e_{t}-e_{t, t-1}^{*}\right)
$$

Obviously (35) and (38) together imply $y_{t}=y_{t}^{f}$. Note that the arbitrariness of the remaining policy parameters means that the policy rule can be expressed in a variety of ways, the most convenient of which is

$$
\mathrm{m}_{\mathrm{t}}=-\alpha_{2} \mathrm{r}_{\mathrm{t}}
$$

This enables the monetary authority to monitor the domestic interest rate, rather than trying to infer exchange rate expectations.

Alternatively, (34) implies that the perfect stability of output will also prevail if

$$
e_{t+1, t}^{*}=\frac{-e_{t-1}^{z} t}{1-\mu_{1}-\mu_{2}}
$$

In general, the expected exchange rate at time $t$, following the observation of the disturbances, is given by (cf (30)),

$$
e_{t+1, t}^{*}=\frac{-\theta t t^{2} t}{1-\mu_{1}-\mu_{2}}
$$


where $\theta_{t}$ is the probability, held at time $t$, of the disturbance at time t being permanent. Thus from (40) and (41) we see that output will be stabilized perfectly about $y_{t}^{f}$ if and only if $\theta_{t}=\theta_{t-1}$, i.e., if and only if agents' perception of the duration of the disturbance does not change between time $t-1$ and $t$. This holds for any intervention rule, including no intervention at all. Essentially, if all disturbances are fully anticipated and the perceived degree of permanence is unchanged, any response in the exchange rate which may occur as a consequence of an arbitrarily chosen intervention policy, will be fully anticipated. Any movements in the domestic price level will hence be perfectly anticipated and output will fluctuate about its frictionless level.

\section{CONCLUSIONS}

The literature on exchange market intervention has restricted the stochastic disturbances impinging on the economy to be purely random white noise. In this paper we have allowed these disturbances to be of more general form. In particular, we have drawn the distinction between disturbances which are unanticipated and anticipated on the one hand, and those that are perceived as being transitory or permanent on the other. In general, we have seen that the appropriate form of exchange market intervention (or more generally money supply rule) is sensitive to these aspects of the disturbances and several interesting conclusions emerge. Disturbances which are unanticipated and perceived as being transitory are the most difficult to stabilize. While the optimal rule turns out to be quite a simple one, involving only the domestic price level and interest rate, the coefficients do depend upon knowing the relative variances of the domestic stochastic disturbances and in general 
it will not succeed in stabilizing output about its frictionless level. By contrast, if the disturbances are perceived as being permanent, then income may be stabilized about its frictionless level by adopting any monetary rule which includes the leaning with the wind component $\mathrm{m}_{\mathrm{t}}=\left(1+\alpha_{2}\right)$ e. The rest of the rule can be specified quite arbitrarily, enabling it to be expressed in a number of convenient, but equivalent, ways. Essentially, the arbitrariness of the rule is due to the appropriate adjustment of the expected exchange rate to each such specification. If all disturbances are perfectly anticipated one period in advance, but are unknown as to whether they are to be transitory or permanent, a simple money supply rule involving the appropriate accomodation to the domestic interest rate will stabilize output perfectly. If both the magnitude and the degree of permanence of the disturbances are known, then any rule--including no intervention-- will provide perfect stability. 


\section{APPENDIX}

\section{Derivation of Supply Function (1e)}

As noted in the text, the supply function is based on the one period wage contract model. We assume that the wage for time $t$ is determined at time 5-1, such that given expectations of firms and workers, the labor market is expected to clear. The expected supply of labor, expressed in logarithms, at the contract wage is

$$
N_{t, t-1}^{S}=n\left(W_{t, t-1}^{c}-P_{t, t-1}^{*}\right)
$$

where

$$
\begin{aligned}
& N_{t, t-1}^{s} \begin{array}{l}
\text { is the expected supply of labor formed at time } \\
t-1 \text { for time } t,
\end{array} \\
& \begin{aligned}
& W_{t, t-1}^{c}= \text { contract wage expressed in logarithms determined } \\
& \text { at time } t-1 \text { for time } t,
\end{aligned} \\
& P_{t}=\begin{array}{l}
\text { price level, expressed in logarithms with } P_{t, t-1}^{*} \\
\text { being its forecast formed in the previous } \\
\text { period. }
\end{array}
\end{aligned}
$$

Assuming further a production function linking the logarithm of output $\mathrm{Y}_{t}$ to the logarithm of employment $\mathrm{N}_{t}$ by

$$
Y_{t}=(1-\theta) N_{t}+\varepsilon_{t}
$$

where $\varepsilon_{t}$ is a stochastic disturbance reflecting technological uncertainty it follows that the expected demand for labor, $\mathrm{N}_{t, t-1}^{\mathrm{d}}$ (based on expected profit maximization) is determined by the marginal product condition

$$
\ln (1-\theta)-\theta N_{t, t-1}^{d}+\varepsilon_{t, t-1}^{*}=W_{t, t-1}^{c}-P_{t, t-1}^{*}
$$


The contract wage is determined by equating the expected demand and supply of labor appearing in (A.1) and (A.3), yielding

$$
W_{t, t-1}^{c}=P_{t, t-1}^{*}+\frac{\ln (1-\theta)}{1+n \theta}+\frac{\varepsilon_{t, t-1}^{*}}{1+n \theta}
$$

and depends positively upon the expected productivity disturbance. Shortrun actual employment is assumed to be determined by the short-run marginal productivity condition for firms after the stochastic variables $\varepsilon_{t}$ and $P_{t}$ are realized, namely

$$
\ln (1-\theta)-\theta N_{t}+\varepsilon_{t}=W_{t, t-1}^{c}-P_{t}
$$

Substituting for $W_{t, t-1}^{c}$ from (A.4) into (A.5) and the resulting expression for $\mathrm{N}_{t}$ into the production function (A.2) yields the supply function

$$
Y_{t}=\frac{(1-\theta) n \ln (1-\theta)}{1+n \theta}+\left(\frac{1-\theta}{\theta}\right)\left(P_{t}-P_{t, t-1}^{*}\right)+\frac{\varepsilon_{t}}{\theta}-\left(\frac{1-\theta}{\theta}\right) \frac{\varepsilon_{t, t-1}^{*}}{1+n \theta}
$$

Letting

$$
\gamma \equiv(1-\theta) / \theta \quad v_{t} \equiv \varepsilon_{t} / \theta
$$

and letting lower case letters measure variables about a nonstochastic level, leads to

$$
y_{t}=\gamma\left(p_{t}-p_{t, t-1}^{*}\right)+v_{t}-\frac{\gamma}{1+n+\gamma} v_{t, t-1}^{*}
$$

which is precisely (le) of the text. 


\section{FOOTNOTES}

*We wish to thank two anonymous referees for their helpful suggestions.

1/We make the usual assumption that all domestic money is held by domestic residents who also hold no foreign money. We therefore abstract from the possibility of "currency substitution," an issue which is receiving some attention in the international monetary literature.

$\underline{2} /$ The condition $z_{k}^{*}=z_{k}, k \leqq 0$ asserts that current and past values of $z$ are known $\mathrm{k}^{\mathrm{o}}$ time $\mathrm{k}^{\prime}$.

3/ In addition, the general solutions for $e^{*}$ include a term containing an arbitrary constant $A_{\text {o }}$ say. This reflects the nonuniqueness of rational expectations solutions. In the case of (5a), A must be set to zero to ensure that the solution is stable. In the case of (5b), however, stability considerations alone do not suffice to determine $A_{0}$. This can be determined only by invoking some additional restriction. In Turnovsky (1983), the procedure was adopted of choosing $A_{0}$ so as to minimize the one period variance of the exchange rate. This approach, which is a variant of the procedure proposed by Taylor (1977), turns out to make the subsequent determination of the optimal intervention policy rather complicated. In the present analysis we invoke the "minimum state representation" procedure proposed recently by McCallum (1983). This involves picking the rational expectations solution based on the simplest soltuion and means that $A_{0}=0$, quite apart from stability considerations. The notion that solutions are based on minimum information is attractive in that it embodies the idea that forecasters use scarce information efficiently.

4/ The case where expectations are backward looking, while consistent with rational expectations, is of less economic interest. In the cases we discuss, the expectations are always forward looking.

5/ In fact, in this case exchange rate expectations are uniform for all future periods. However, since only $e^{*}+1, t$ appears in the model, this is the only expression we need consider.

6/ For a one period transitory disturbance, we find that $e_{t+i, t}^{*}=0$ for $i=2,3, \ldots$ There is also a backward looking solution, which is discarded in favor of the forward looking solution, which is not only of greater economic interest, but also for a transitory disturbance is always finite for all values of $\mu_{1}, \mu_{2}$.

I'See, e.g., the discussion in the survey by Marston (1983).

8/ It is worth nothing that this result therefore also obtains for the more usual restrictive type of intervention rule which utilizes only information on the current exchange rate. 
$\underline{9} /$ Substituting (30) into (14a) yields

$$
y^{t}=y_{t}^{f}+\frac{\gamma}{\Delta}\left[1+\alpha_{2}-\mu_{1}\right]\left[\frac{v_{t}}{1+n+\gamma}-\frac{z_{t}}{1-\mu_{1}-\mu_{2}}+q_{t}\right]+\frac{\gamma\left(\alpha_{2}+\mu_{2}\right)(1-\theta) z_{t}}{\Delta\left(1-\mu_{1}-\mu_{2}\right)}
$$

Note that it would appear that $y_{t}$ can be stabilized perfectly about $y_{t}^{f}$ by setting $\mu_{1}=1+\alpha_{2}, \mu_{2}=-\alpha \frac{t}{2}$. However, this is infeasible since for these values of the policy parameters, the money market equilibrium condition reduces to

$$
\left(\alpha_{2}+\mu_{3}\right) \omega_{t}-\left(1-\mu_{4}\right) q_{t}-u_{t}=0
$$

which with $u_{t}$, $w_{t}$ and $q_{t}$ being independently distributed random variables generafly does not hold. 


\section{REFERENCES}

Barro, R., 1976, "Rational Expectations and the Role of Monetary Policy," Journal of Monetary Economics, 2, 1-32.

Boyer, R., 1978, "Optimal Foreign Exchange Market Intervention," Journal of Political Economy, 86, 1045-1056.

Cox, W. M., 1980, "Unanticipated Money,Output, and Prices in the Small Economy," Journal of Monetary Economics, 6, 359-384.

Fischer, S., 1977, "Wage Indexation and Macroeconomic Stability," in K. Brunner and A. Meltzer, eds., Stabilization of the Domestic and International Economy, (North-Holland, Amsterdam).

Frenke1, J. A. and J. Aizenman, 1982, "Aspects of the Optimal Management of Exchange Rates," Journal of International Economics, 13, 231-256.

Gray, J., 1976, "Wage Indexation: A Macroeconomic Approach," Journal of Monetary Economics, 2, 221-235.

Henderson, D. W., 1979, "Financial Policies in Open Economics," American Economic Review, Papers and Proceedings, 69, 232-239.

Marston, R. C., 1983, "Stabilization Policies in Open Economies," in R. W. Jones and P. B. Kenen (eds.), Handbook of International Economics, (North-Holland, Amsterdam).

McCallum, B. T., 1983, "On Non-uniqueness in Rational Expectations Models: An Attempt at Perspective," Journal of Monetary Economics, 11, 139-168.

Poole, W., 1970, "Optimal Choice of Monetary Policy Instruments in a Simple Stochastic Macro Model," Quarterly Journal of Economics, $84,197-216$.

Roper, D. E. and S. J. Turnovsky, 1980, "Optimal Exchange Market Intervention in a Simple Stochastic Macro Model," Canadian Journal of Economics, 13, 296-309.

Taylor, J., 1977, "Conditions for Unique Solutions in Stochastic Macroeconomic Models with Rational Expectations," Econometrica, 45, 1377-1385.

Turnovsky, S. J., 1983, "Exchange Market Intervention in a Small Open Economy," in.J. Bhandari and B. Putnam (eds.), The International Transmission of Economic Disturbances, (MIT Press, Cambridge).

Turnovsky, S. J., 1984, "Optimal Exchange Market Intervention: Two Alternative Classes of Rules," in J. Bhandari (ed.), Exchange Rate Management Under Uncertainty, (MIT Press, Cambridge). 